\title{
MEIS1 down-regulation by MYC mediates prostate cancer development through elevated HOXB13 expression and AR activity
}

\author{
Nichelle C. Whitlock ${ }^{1}$. Shana Y. Trostel ${ }^{1} \cdot$ Scott Wilkinson $\mathbb{D}^{1} \cdot$ Nicholas T. Terrigino $^{1} \cdot$ S. Thomas Hennigan ${ }^{1}$. \\ Ross Lake ${ }^{1} \cdot$ Nicole V. Carrabba $^{1} \cdot$ Rayann Atway $^{1} \cdot$ Elizabeth D. Walton ${ }^{1} \cdot$ Berkley E. Gryder $\mathbb{B}^{2} \cdot$ Brian J. Capaldo $^{1}$. \\ Huihui $\mathrm{Ye}^{3,4} \cdot$ Adam G. Sowalsky (iD)
}

Received: 16 March 2020 / Revised: 29 June 2020 / Accepted: 3 July 2020 / Published online: 17 July 2020

(c) The Author(s) 2020. This article is published with open access

\begin{abstract}
Localized prostate cancer develops very slowly in most men, with the androgen receptor (AR) and MYC transcription factors amongst the most well-characterized drivers of prostate tumorigenesis. Canonically, MYC up-regulation in luminal prostate cancer cells functions to oppose the terminally differentiating effects of AR. However, the effects of MYC upregulation are pleiotropic and inconsistent with a poorly proliferative phenotype. Here we show that increased MYC expression and activity are associated with the down-regulation of MEIS1, a HOX-family transcription factor. Using RNAseq to profile a series of human prostate cancer specimens laser capture microdissected on the basis of MYC immunohistochemistry, MYC activity, and MEIS1 expression were inversely correlated. Knockdown of MYC expression in prostate cancer cells increased the expression of MEIS1 and increased the occupancy of MYC at the MEIS1 locus. Finally, we show in laser capture microdissected human prostate cancer samples and the prostate TCGA cohort that MEIS1 expression is inversely proportional to AR activity as well as HOXB13, a known interacting protein of both AR and MEIS1. Collectively, our data demonstrate that elevated MYC in a subset of primary prostate cancers functions in a negative role in regulating MEIS1 expression, and that this down-regulation may contribute to MYC-driven development and progression.
\end{abstract}

\section{Introduction}

Locally advanced prostate cancers harbor a limited number of recurrently altered genes whose expression change at the earliest stages of tumor development. These include downregulation of the tumor suppressors NKX3-1 and PTEN (often

Supplementary information The online version of this article (https:// doi.org/10.1038/s41388-020-01389-7) contains supplementary material, which is available to authorized users.

Adam G. Sowalsky

adam.sowalsky@nih.gov

1 Laboratory of Genitourinary Cancer Pathogenesis, National Cancer Institute, NIH, Bethesda, MD 20892, USA

2 Genetics Branch, National Cancer Institute, NIH, Bethesda, MD 20892, USA

3 Department of Pathology, Beth Israel Deaconess Medical Center, Boston, MA 02215, USA

4 Present address: Department of Pathology, University of California Los Angeles, Los Angeles, CA 90095, USA due to genomic deletion), up-regulation of $E R G$ (due to fusion with TMPRSS2), and up-regulation of $M Y C$, which often cooccurs with a single-copy gain of chromosome 8q24 [1-5]. Although up-regulation of $M Y C$ in most neoplastic tissues is a very early event that contributes to self-renewal and proliferation, localized prostate cancer (PCa) is not proportionally as hyperproliferative as metastatic prostate cancers which frequently harbor focal amplification of $M Y C[6,7]$. In part, the effects of the androgen receptor (AR) in terminallydifferentiated luminal prostate cells are disrupted by MYC and other co-factors including FOXA1 and HOXB13 to reengage proliferative processes during tumorigenesis [7-11].

Recently, increased awareness that the vast majority of prostate cancers are indolent has led to increased molecular profiling of tumor biopsies prior to definitive treatment. Although MYC expression has been observed in the cancer precursor high-grade prostatic intraepithelial neoplasia, MYC expression in indolent-appearing tumor cells predicts the presence of higher-grade disease and is associated with poor differentiation [3-5]. In localized prostate cancers, up-regulated MYC has been further associated with alterations in nucleoli structure, concomitant with increased biogenesis of ribosomal 
A

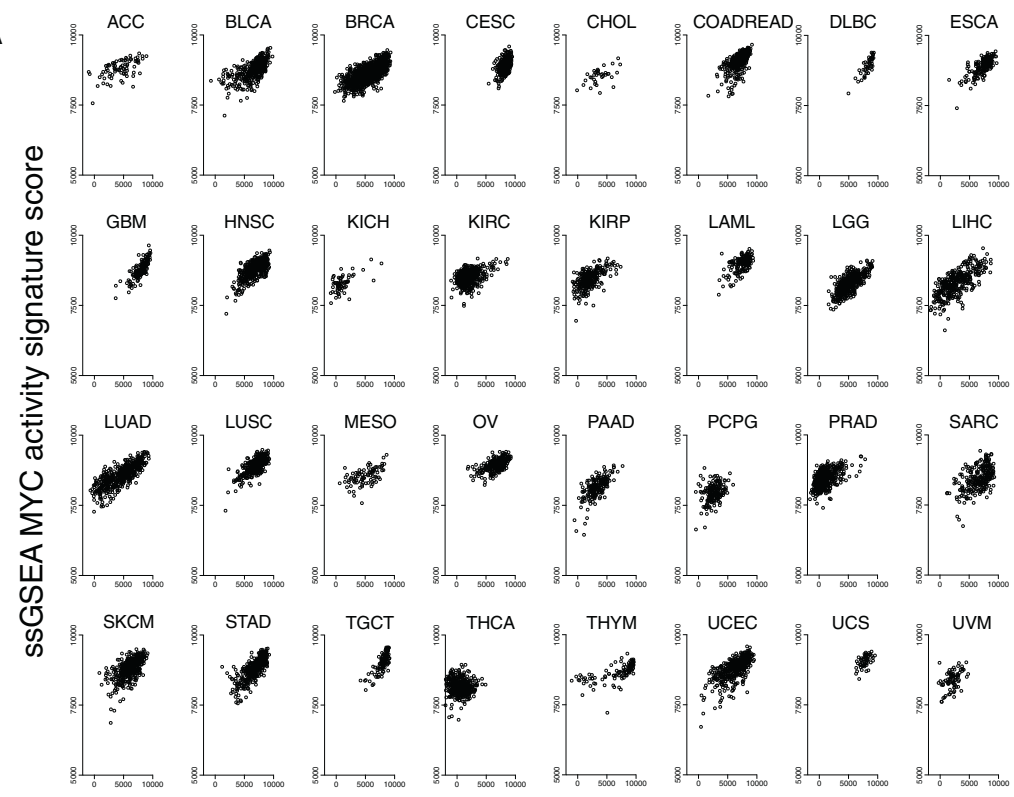

ssGSEA proliferation signature score

B

BHC

MYC

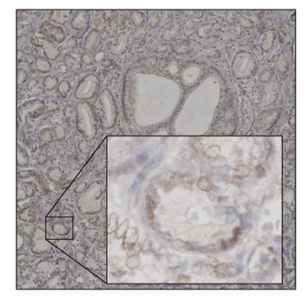

Ki67

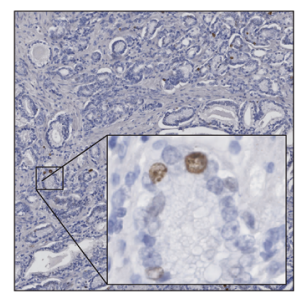

C

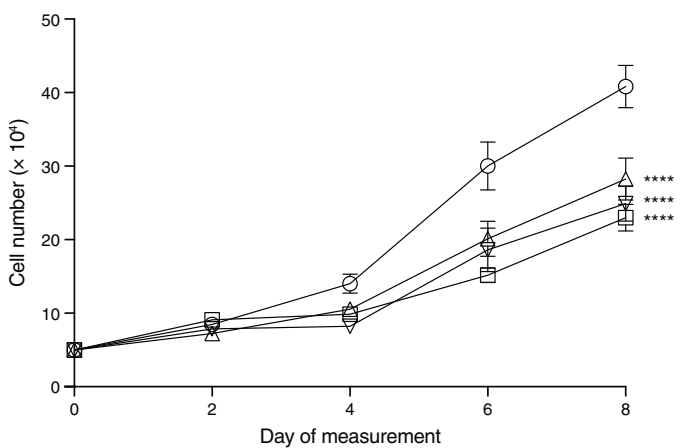

$\bigcirc$ LNCaP/NTC $\square$ LNCaP/MYC ${ }^{512} \triangle$ LNCaP/MYC $^{627} \quad \nabla \quad$ LNCaP/MYC $^{637}$

RNA, increased purine metabolism, and expression of the telomere RNA subunit TERC [12-14]. These effects contrast sharply with the phenotype of highly amplified MYC, which is enriched in metastatic and treatment-resistant prostate cancers,
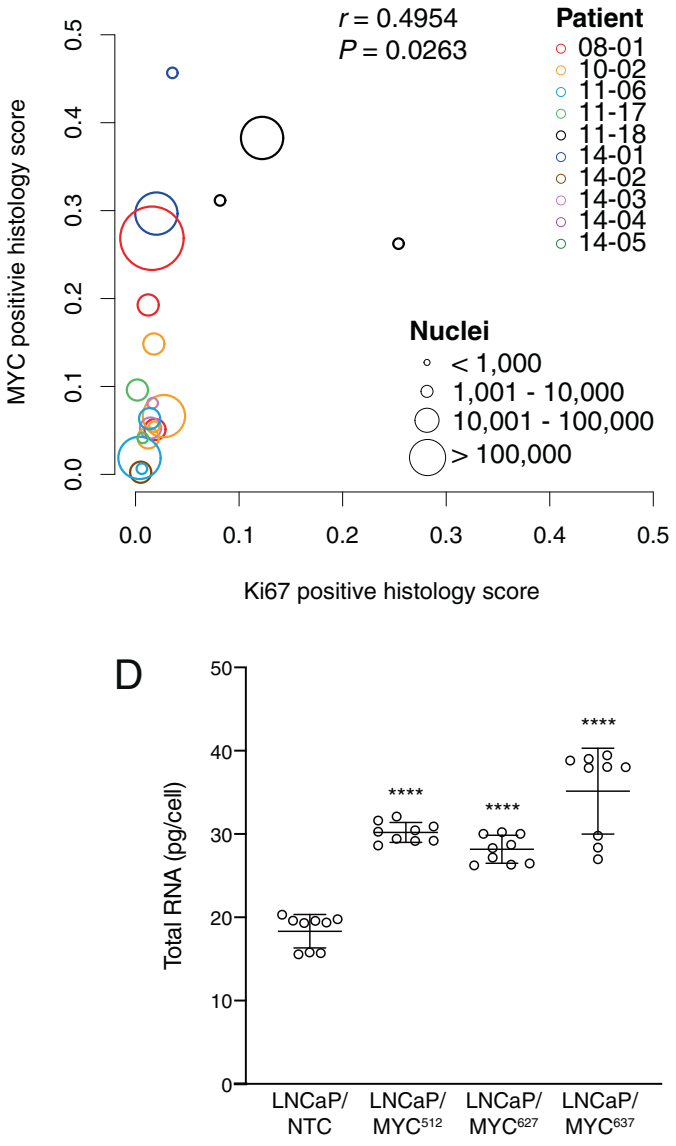

and mirrors the role of MYC in other cancer types via effects on AKT to contribute toward cell division and survival [15-17]. Nonetheless, even in primary PCa, MYC protein expression is diffuse and heterogeneous [3]. 
Fig. 1 MYC and proliferation in human cancers. a Left: correlation of the 54-gene ssGSEA MYC activity signature score and the 17-gene ssGSEA proliferation signature score for each tumor type from the TCGA pan-cancer cohort. All scatter plots are on the same scale. Right: plot of Pearson $r$ coefficients of correlation from each scatter plot shown on the left. All tumor types showed a positive correlation, ranging from 0.3386 (PCPG: pheochromocytoma and paraganglioma) to 0.8473 (DLBC: diffuse large B-cell lymphoma). PRAD: prostate adenocarcinoma, $r=0.4822$. All correlations were significant at $P<$ 0.001 except THCA (thyroid carcinoma; $P=0.5543$ ). b Left: representative IHC staining used as input for automated image analysis with Definiens Tissue Studio and the processed file used for quantification of histologic score via nuclear classification, for anti-MYC and antiKi67 immunostains. Right: unweighted scatter plot and Pearson correlation of the quantified intensities of anti-Ki67 vs. anti-MYC stain. Each open circle represents a contiguous quantified region, colorcoded by patient. Multiple regions from the same patient indicate multiple noncontiguous regions were measured, and the size of each circle indicates the number of nuclei considered for the analysis. c Assessment of cell growth in vitro as a function of $M Y C$ knockdown. LNCaP cells expressing non-targeting or MYC-targeting hairpins were seeded at a density of 50,000 cells per well and counted every 2 days in triplicate using a hemocytometer by three different individuals blinded to the identities of the cultures. Data shown represents the mean \pm standard error of ten independent experiments. $* * * * P<0.0001$ for each cell line vs. control by two-way ANOVA. d RNA content as a function of $M Y C$ knockdown. Total RNA was extracted from $1 \times 10^{6}$ pelleted LNCaP cells expressing non-targeting or $M Y C$-targeting hairpins. RNA concentrations were measured by Nanodrop and total RNA was calculated per cell. Data represents mean \pm standard deviation of three independent experiments conducted in triplicate, with open circles representing individual replicate RNA quantity values $(* * * P<0.0001$ for each cell line vs. control by two-way ANOVA).

We sought to examine the genetic contribution of MYC to the development and progression of primary $\mathrm{PCa}$ in the context of dysregulated growth by using anti-MYC immunohistochemistry (IHC) and performing laser capture microdissection on populations of human prostate tumor cells with varying expression of MYC protein. We show using transcriptome profiling that increased MYC activity is inversely correlated with expression of Myeloid Ecotropic viral Insertion Site 1 (MEIS1), a transcription factor that interacts with and regulates the activity of HOX homeodomain transcription factors, including HOXB13 [18-20]. We determined that MYC binding to the MEIS1 locus decreases as MYC levels increase, and that MEISI expression is negatively correlated with $H O X B 13$ expression and AR activity.

\section{Results}

\section{MYC activity is weakly associated with proliferation in primary prostate cancer}

Although MYC orchestrates a broad range of biological functions, it has been shown in many cancers that MYC drives tumorigenesis by potentiating or stimulating cell growth and proliferation [21]. To assess the relationship between MYC activity and proliferation across cancer types, we evaluated MYC activity and cell proliferation rate in TCGA pan-cancer cohort using ssGSEA with MYC activity and proliferation signatures [22]. As shown in Fig. 1a, signatures for MYC activity and proliferation were positively correlated in all cancers with the vast majority showing strong positive correlations $(r>0.5)$. However, prostate adenocarcinoma (PRAD) was below this cut-off as one of the weakest correlating cancer types $(r=0.4822$, $95 \%$ C.I. $0.4112-0.5472, P<0.0001)$. Repeating this analysis with a prostate cancer-specific gene set generated by MYC overexpression in LNCaP cells [11], PRAD correlation with proliferation increased modestly $(r=0.5609,95 \%$ C.I. $0.4972-0.6186, P<0.0001$ ), but was still below the median correlation coefficient (Supplementary Fig. 1). To validate this observation, we screened a new cohort of treatment naïve Gleason score 7 prostate tumors for MYC and Ki67 expression by IHC. Although MYC was expressed in a majority of tumor cells, Ki67 expression was limited to $<5 \%$ of cells (Fig. 1b, left), consistent with median Ki67 indices from large cohorts of GS7 tumors in the 2-4\% range $[23,24]$. We then applied automated image analysis to quantify millions of nuclei across 20 tumor foci from ten different cases. While the MYC positive histology score ranged from 0 (low expression) to 0.46 (high expression), the Ki67 positive histology score was $<0.1$ in 18 out of 20 foci (Fig. 1b, right). An unweighted Pearson correlation demonstrated that MYC IHC was weakly but positively correlated with Ki67 $(r=0.4954$, 95\% C.I. $0.06772-0.7693, P=0.0263$ ), similar to what we observed in the PRAD TCGA cohort, indicating that the transcriptional signatures applied to the larger dataset accurately recapitulated MYC and Ki67 protein abundance. Because high Ki67 expression was not associated with high MYC expression, proliferation may have been dependent upon MYC activity, but MYC expression alone was not sufficient for proliferation.

To further examine the role of MYC in prostate tumorigenesis, we knocked down MYC in LNCaP cells, which had physiologically-elevated levels of MYC. Like primary $\mathrm{PCa}, \mathrm{LNCaP}$ cells express AR, are androgen sensitive, and grow slowly despite elevated MYC [25]. Using MYCknockdown cells as a model of MYC-low PCa cells, we show in Supplementary Fig. 2 that we achieved $~ 50 \%$ reduction in $M Y C$ transcript and MYC protein expression. As anticipated, knockdown of $M Y C$ was associated with a modest decrease in cell number at each time point (Fig. 1c) but the decrease in cell proliferation was not proportional to the decrease in $M Y C$ expression. The relationship between MYC and proliferation in other cancer types also stipulates a role for MYC as a universal amplifier of transcription [26], alleviating constraints on cell growth and proliferation 
[27]. However, we observed the opposite: the total amount of cellular RNA increased when $M Y C$ was knocked down in LNCaP cells (Fig. 1d). Together, these findings indicate that the cellular behavior of MYC in PCa contrasts with other tumor types, and that MYC does not act solely in a proliferative capacity.

\section{Increased MYC is associated with specific up- and down-regulation of target genes}

We hypothesized that analysis of tumors with different levels of MYC expression would identify genes that may contribute to MYC activity in PCa pathogenesis. Using samples of human radical prostatectomy specimens stained with anti-MYC, we identified concomitant regions of highand low-MYC expression in the same patients. We subjected these within-patient sets of tumor foci to laser capture microdissection (Fig. 2a), controlling for PTEN and ERG status by confirming their concordance within each case. Of the 19 foci microdissected, we designated 42\% MYC-high $(n=8)$ and 58\% MYC-low $(n=11)$. We performed RNAseq on these cases and derived a limited gene set of 293 upor down-regulated genes, with only 15 genes showing expression changes of fourfold or more with adjusted $P$ values $<0.10$ (Fig. $2 \mathrm{~b}$ and Supplementary Table 2). Consistent with our observation of increased RNA in LNCaP cells expressing MYC knockdown hairpins (see Fig. 1d), we observed more genes down-regulated than up-regulated in MYC-high vs. MYC-low tumor foci.

To further refine genes of potential interest, we analyzed a second dataset comprised of whole transcriptome sequencing from 499 primary tumors in the TCGA PRAD cohort. Using the mRNA expression values of genes in the MYC activity signature (see Fig. 1a and Supplementary Table 1), we compared the top 20 and bottom 20 ranked cases based on average median absolute deviation-modified $z$-score (Supplementary Fig. 3), and established a second list of differentially expressed genes (Fig. 2c and Supplementary Table 3). As these cases were compared between patients rather than within-patient, a far greater number of genes were differentially expressed (1346) with fold-change of at least 4 and adjusted $P$ values $<0.05$.

When comparing differentially expressed genes between MYC-high vs. MYC-low tumors, MYC expression was consistently greater in the MYC-high group in the LCM primary PCa cohort (Fig. 2b and Supplementary Table 2). As MYC RNA and protein expression often do not correlate due to the tight posttranslational regulation of MYC $[28,29]$, the statistically insignificant increase of $M Y C$ expression in the MYC-high group was not unexpected. However, MYC was amongst the up-regulated genes in the MYC activity high group, confirming proper stratification in the TCGA PRAD cohort as well (Fig. 2c and
Supplementary Table 3). Taking an inclusive approach to combine both datasets, we identified 293 and 7390 differentially expressed genes between MYC-high and MYC-low prostate tumors in the LCM tissue and TCGA PRAD cohorts, respectively (Fig. 2d, e) that were concordantly regulated in the same direction. Of these, 221 were in common at a false discovery rate threshold of 0.10 for LCM tissue and 0.05 for TCGA PRAD (Fig. 2e and Supplementary Table 4).

\section{MEIS1 is negatively associated with MYC expression and MYC activity}

Given that we observed more genes down-regulated in MYC-high tumors than we would have expected if MYC were functioning as a genome-wide transcriptional amplifier, we hypothesized that collective analyses of gene expression might reveal coordinated down-regulation of biological processes that may contribute to MYC-driven $\mathrm{PCa}$ tumorigenesis. We therefore performed gene set enrichment analysis (GSEA) using both the LCM and TCGA cohort datasets as inputs, limiting our analyses to gene sets with differentially expressed genes shared by the LCM and TCGA cohorts. As depicted in Fig. 3a, our initial comparative analyses demonstrated significant and concordant enrichment of 762 gene sets (Supplementary Table 5). To narrow our focus further, we refined our search only to include gene sets based on ChIP-seq target discovery studies with the premise that increased MYC activity would misregulate a multitude of transcriptionally-driven pathways. These analyses identified 11 gene sets, a preponderance of which were associated with development and survival (Table 1).

Of note, gene sets related to NOTCH, RUNX1, HOXA9, and TNF were negatively enriched in MYC-high vs. MYClow tumors. This suggested that one or more transcription factors exert effects in opposition to MYC, and may be guiding MYC-mediated misregulation of these pathways. In seeking a gene or common regulator between these gene sets, we identified the transcription factor MEIS1 as an important regulator in each of the aforementioned pathways. For example, MEIS1 has been shown to regulate genes in the NOTCH pathway [30] and sensitizes cells to TNF [31]. Moreover, MEIS1 is essential for the expression of genes driven by the HOXA9-NUP98 fusion in acute myeloid leukemia [32-34]. Therefore, we examined whether MEISI expression was associated with increased MYC activity as a proxy for its role in $\mathrm{PCa}$ development.

We observed that MEISI expression was reduced in MYChigh cases compared with MYC-low tumors, for both the LCM and TCGA cohorts (Supplementary Tables 2 and 3, respectively). At a case-by-case level, MEISI expression was negatively correlated with MYC (Fig. $3 b ; r=-0.4321,95 \%$ 


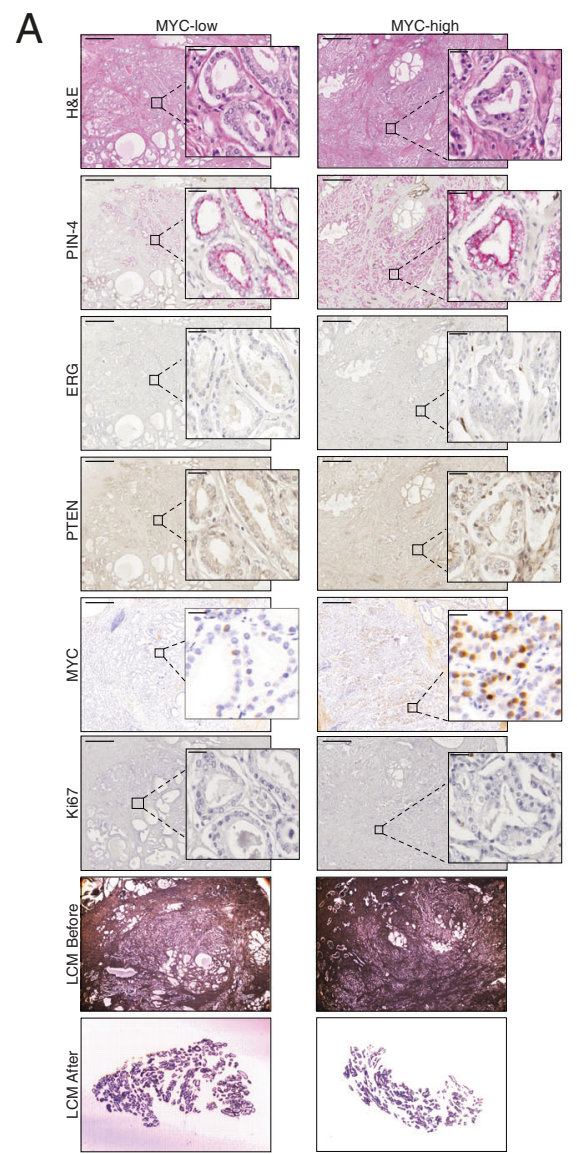

Fig. 2 Effect of MYC levels on gene expression. a Representative IHC of case 14-03 showing heterogeneity of MYC expression within a single patient. Serial sections were stained with H\&E and PIN-4 cocktail to distinguish benign glands and high grade prostatic intraepithelial neoplasia from tumor foci; anti-ERG and anti-PTEN immunostains were performed to select regions with concordant phenotypes. Micrographs of stained tissue before and after laser capture microdissection (LCM) are also shown. Scale bar: $500 \mu \mathrm{m}$; inset scale bar: $20 \mu \mathrm{m}$. b Volcano plot depicting differentially expressed genes in MYC-high vs. MYC-low tumors in the LCM cohort, with the false discovery rate (adjusted $-\log _{10} P$ value) on the $y$-axis and the $\log _{2^{-}}$ transformed fold-change (MYC-high vs. MYC-low) on the $x$-axis. Expression of $M Y C$ is shown in red. Black dots represent genes with

C.I. $-0.7409-0.0275, P=0.0323)$ and MYC activity (Fig. 3c; $r=-0.5527,95 \%$ C.I. -0.7372 to -0.2713 , $P=0.0001)$ in the LCM and TCGA cohorts, respectively. To assess whether this association occurred in unselected populations of primary $\mathrm{PCa}$, we analyzed RNA-seq data from an additional 69 microdissected tumor foci and the entire TCGA PRAD cohort $(n=499)$. Although weaker than the MYCselected cohorts, the negative relationship between MEIS and $M Y C$ expression was still observed (Fig. 3d, e).

\section{MYC negatively regulates MEIS1 expression}

Recently, Bhanvadia et al., postulated that higher MEISI expression conferred a less aggressive PCa phenotype [19]. fold-change $\geq \pm 4$ and adjusted $P$ value $<0.10$ for Welch's $t$ test. c Volcano plot depicting differentially expressed genes in MYC activity high vs. MYC activity low tumors in the TCGA PRAD cohort, with the false discovery rate (adjusted $-\log _{10} P$ value) on the $y$-axis and the $\log _{2}$-transformed fold-change (MYC activity high vs. MYC activity low) on the $x$-axis. Expression of $M Y C$ is shown in red. Black dots represent genes with fold-change $\geq \pm 4$ and adjusted $P$ value $<0.05$ for Welch's $t$ test. d Flowchart of the strategy used to identify lists of differentially expressed genes from the LCM and TCGA PRAD cohorts. e Euler diagram depicting distinct and shared differentially expressed genes between the LCM and TCGA PRAD cohort. Circles are drawn to scale.

Based on these results, we hypothesized that repression of MEIS1 expression by MYC may contribute to the aggressivity of MYC-driven PCa. We engineered $\mathrm{LNCaP}$ cells with a non-targeting hairpin as MYC-high and LNCaP cells with three different $M Y C$-targeting hairpins as MYC-low (see Supplementary Fig. 2), with variable efficiency of MYC knockdown to replicate the biological variability observed in patient samples. We then performed ChIP-seq against MYC in these cells to generate genome-wide site maps and ascertain chromatin occupancy at MEISI. In these cells, we had observed increased overall transcriptional output (see Fig. 1d), and we controlled for this phenomenon using Drosophila chromatin spike-in controls. 


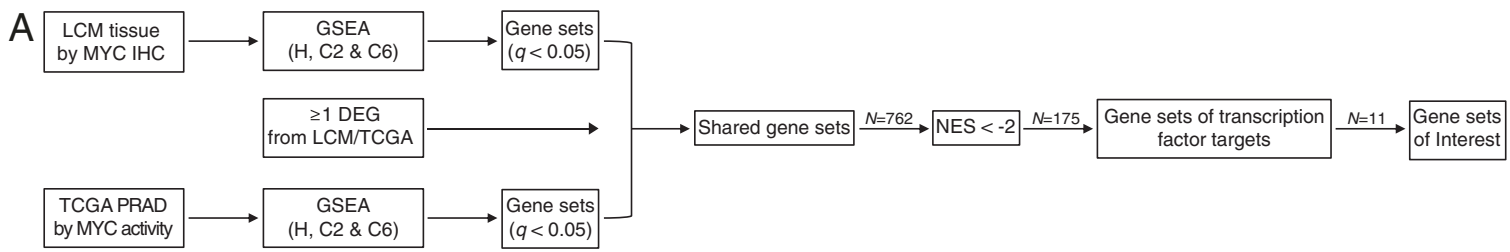

B

LCM of tissue by MYC IHC $(N=19)$

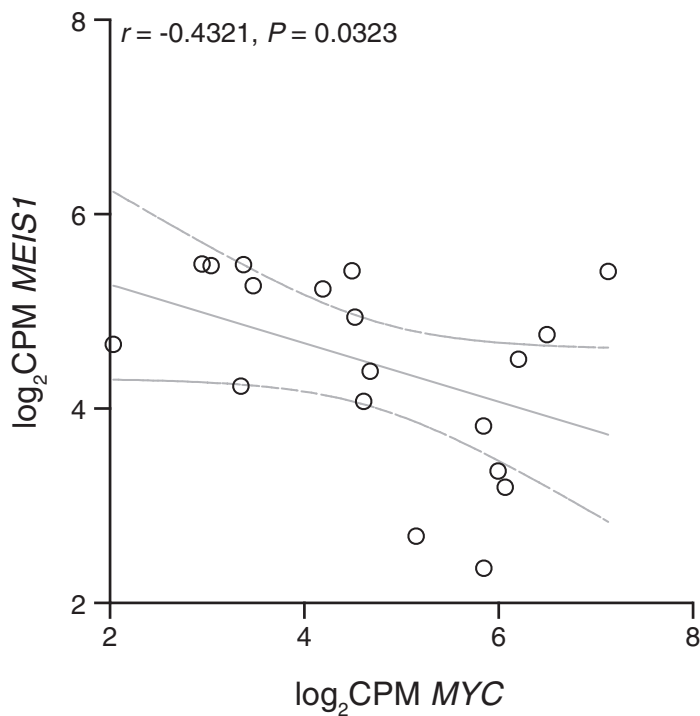

D

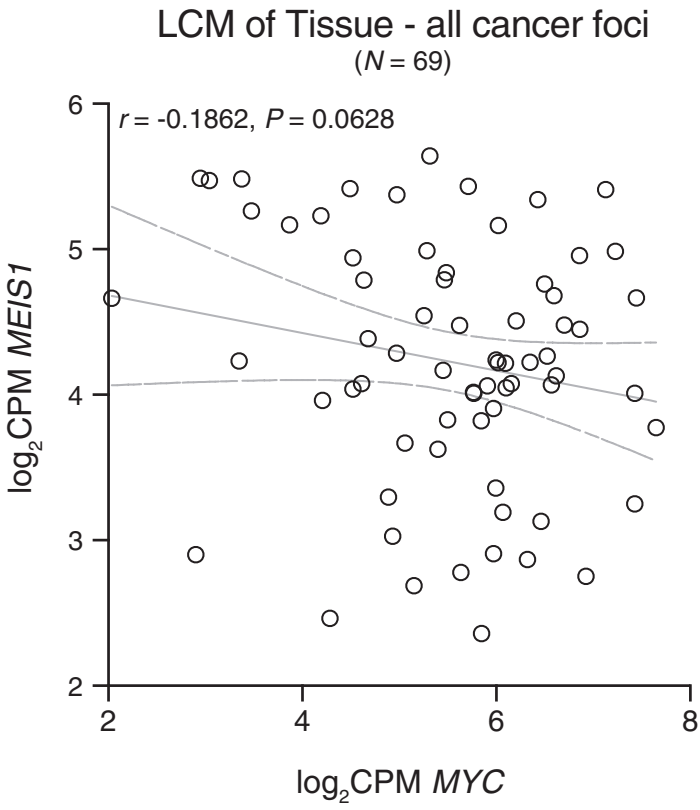

Fig. 3 Association between MYC and MEIS1 expression. a Flowchart of the strategy used to identify potential MYC target pathways based on differentially expressed genes. b-e Correlation of the $\log _{2}$ CPM expression levels between MYC and MEISI in the initial LCM

The quality of our MYC ChIP DNA was assessed using Active Motif's ChIP-IT qPCR Analysis Kit and primers targeting a MYC super-enhancer region prior to sequencing. As shown in Supplementary Fig. 4A, an enrichment of six-
C

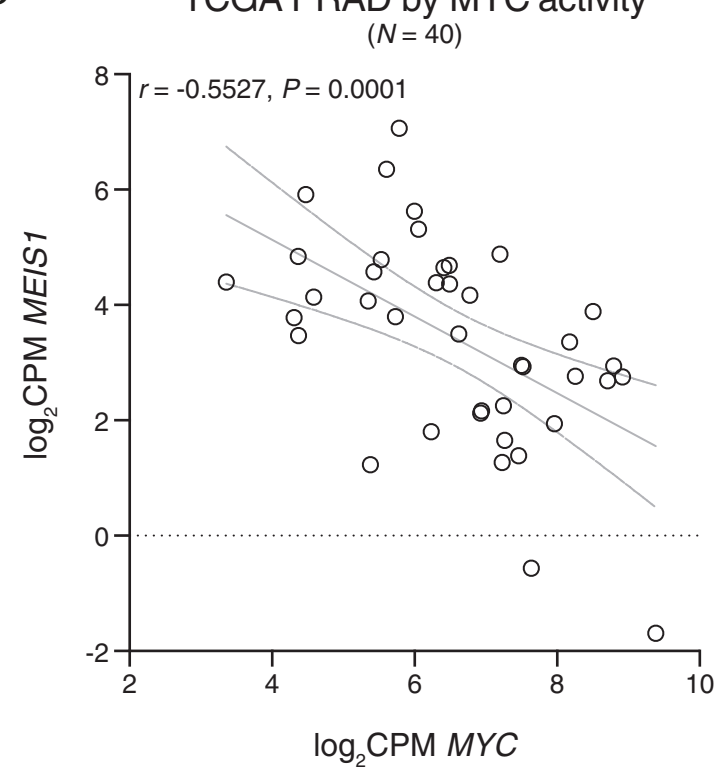

E TCGA PRAD - all cases $(N=499)$

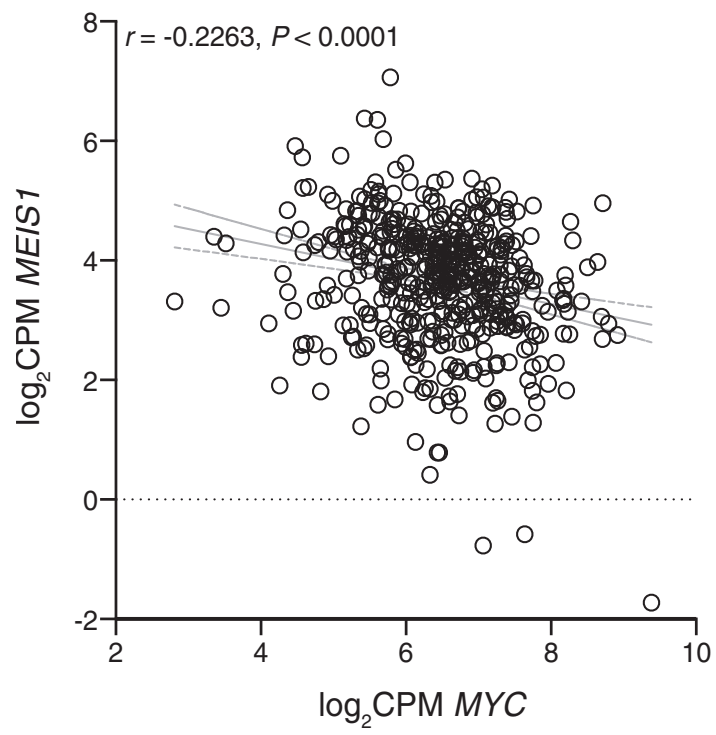

cohort $(N=19, \mathbf{b})$, the initial TCGA PRAD cohort $(N=40, \mathbf{c})$, the entire LCM primary PCa cohort $(N=69, \mathbf{d})$, and the entire TCGA PRAD cohort $(N=499$, e). The gray dotted lines represent the $95 \%$ confidence intervals for each correlation.

to eight-fold was achieved for each experiment and thus submitted for subsequent ChIP-seq analysis. We identified $\sim 18,000$ and 27,000 high confidence peaks $\left(P<1 \times 10^{-9}\right)$ in $\mathrm{LNCaP} / \mathrm{NTC}$ and $\mathrm{LNCaP} / \mathrm{shMYC}$ consensus sites (that is, 
Table 1 List of common gene sets negatively enriched in MYC-activity high vs. low comparing LCM tissue and TCGA cohorts.

\begin{tabular}{|c|c|c|c|c|}
\hline \multirow[b]{2}{*}{ MSigDB gene set } & \multicolumn{2}{|c|}{ LCM tissue } & \multicolumn{2}{|l|}{ TCGA } \\
\hline & NES & $Q$ value & NES & $Q$ value \\
\hline Onder CDH1 Targets 3 DN & -2.041 & 4.27E-03 & -2.193 & $6.96 \mathrm{E}-03$ \\
\hline Ono AML1 Targets DN & -2.154 & $2.18 \mathrm{E}-03$ & -2.135 & $6.96 \mathrm{E}-03$ \\
\hline Klein Targets of BCR ABL1 Fusion & -2.192 & $1.80 \mathrm{E}-03$ & -2.274 & $6.96 \mathrm{E}-03$ \\
\hline Hess Targets of HOXA9 and MEIS1 DN & -2.206 & $1.63 \mathrm{E}-03$ & -2.192 & $6.96 \mathrm{E}-03$ \\
\hline Takeda Targets of NUP98 HOXA9 Fusion DN & -2.216 & $1.43 \mathrm{E}-03$ & -2.005 & $6.96 \mathrm{E}-03$ \\
\hline Phong TNF Targets UP & -2.222 & $1.41 \mathrm{E}-03$ & -2.207 & $6.96 \mathrm{E}-03$ \\
\hline Kim GLIS2 Targets UP & -2.431 & $1.75 \mathrm{E}-04$ & -2.066 & $6.96 \mathrm{E}-03$ \\
\hline Hecker IFNB1 Targets & -2.497 & $1.50 \mathrm{E}-04$ & -2.086 & $6.96 \mathrm{E}-03$ \\
\hline Servitja Islet HNF1A Targets UP & -2.542 & $8.51 \mathrm{E}-05$ & -2.164 & $6.96 \mathrm{E}-03$ \\
\hline Vilmas NOTCH1 Targets UP & -2.575 & $8.93 \mathrm{E}-05$ & -2.342 & $6.96 \mathrm{E}-03$ \\
\hline Gaurnier PSMD4 Targets & -2.768 & $0.00 \mathrm{E}+00$ & -2.311 & $6.96 \mathrm{E}-03$ \\
\hline
\end{tabular}

NES normalized enrichment score. sites common in all three $M Y C$ knockdown lines), respectively; and of these, $98 \%$ of all peaks were shared (Supplementary Fig. 4B). These sites overlapped with those reported in multiple publicly-available MYC ChIP-seq datasets $([11,35-37])$, with the extent of overlap ranging from 22 to $90 \%$ (Supplementary Table 6). Of note, when compared with another LNCaP MYC ChIP-seq, the overlap ranged from 77 to $90 \%$, validating the technical results of our experiment. Applying functional annotation of binding sites, the majority of MYC-bound peaks were either promoter proximal or enriched for intronic/intergenic DNA in both control and MYC knockdown cell lines (Supplementary Fig. 4C). This distribution was also similar to that of previous MYC ChIP-seq datasets, providing orthogonal validation of our data (Supplementary Fig. 4D).

When we examined MYC ChIP-seq peaks in the vicinity of MEISI, we observed that proportional MYC recruitment to MEIS1 was increased 1.29-fold on average, relative to global binding in each of the three MYC shRNA lines relative to control (Fig. 4a). In contrast to MEISI, we would anticipate that genes positively regulated by MYC would demonstrate reduced recruitment of MYC at their loci when MYC levels are reduced by shRNA, counter to what we observed in Fig. 4a. When we examined binding events at the known MYC-regulated gene PNP, we observed 1.17fold less MYC recruitment, on average, to the PNP locus (Fig. 4b). For both MEIS1 and PNP, ChIP-seq for H3K27ac marks in parental LNCaP cells also identified a peak at the same genomic position, indicating that the MYC ChIP-seq was identifying chromatin of active enhancer elements. Similar results were observed for two additional MYC targets, NOLCl and EIF5A (Supplementary Fig. 5A-B) of 1.28-fold less binding at each locus. The increased occupancy of MYC at MEISI was far more pronounced when peaks were normalized to Drosophila spike-in controls (Supplementary Fig. 5C), although this also resulted in minimal differences between control and $M Y C$ knockdown for MYC binding at PNP (Supplementary Fig. 5D). Nonetheless, motif enrichment analysis at MYC-ChIP peaks showed decreases at canonical MYC binding sites upon MYC knockdown (Fig. 4c).

We then sought to determine if the observed increase in chromatin occupancy translated to altered MEISI transcription by qRT-PCR. Knockdown of $M Y C$ resulted in increased abundance of the MEIS1 transcript, consistent with the observed increase in MYC recruitment at MEISI (Fig. 4d). Together, these results support the role of MYC in the negative regulation of MEISI in primary PCa.

In PCa, MEIS1 functions to direct transcriptional specificity and activity of HOXB13 and act as a negative regulator of AR [20, 38]. Therefore, we next determined whether $H O X B 13$ expression or AR activity were altered in the context of MYC activity. Indeed, there were statistically significant positive correlations between MYC and HOXB13 mRNA levels in both the entire LCM tissue cohort (Fig. 4e, top; $r=0.6801,95 \%$ C.I. $0.5285-0.7897, P<0.0001)$ and the entire TCGA PRAD cohort (Fig. 4f, top; $r=0.0894$, 95\% C.I. 0.0016-0.1758, $P=0.0230$ ) although the strength of correlation in the TCGA cases was notably less than the purer LCM tissue cohort. We also observed a positive correlation between MYC mRNA levels and the ssGSEA scores for AR activity [39] in both the LCM cohort (Fig. 4e, bottom; $r=0.7550,95 \%$ C.I. $0.6311-0.8413, P<0.0001$ ) and the TCGA cohort (Fig. 4f, bottom; $r=0.0567,95 \%$ C. I. $-0.0312-0.1438, P=0.1029)$, although statistical significance was reached solely in the LCM cohort. Moreover, there was a statistically significant negative correlation between MEISI and HOXB13 expression in both the LCM cohort (Fig. 4g, top; $r=-0.4495$, 95\% C.I. -0.6202 to $-0.2381, P<0.0001$ ) and the TCGA cohort (Fig. 4h, top; $r=-0.5273,95 \%$ C.I. -0.5879 to $-0.4608, P<0.0001$ ). This inverse association was also observed between MEISI 
A
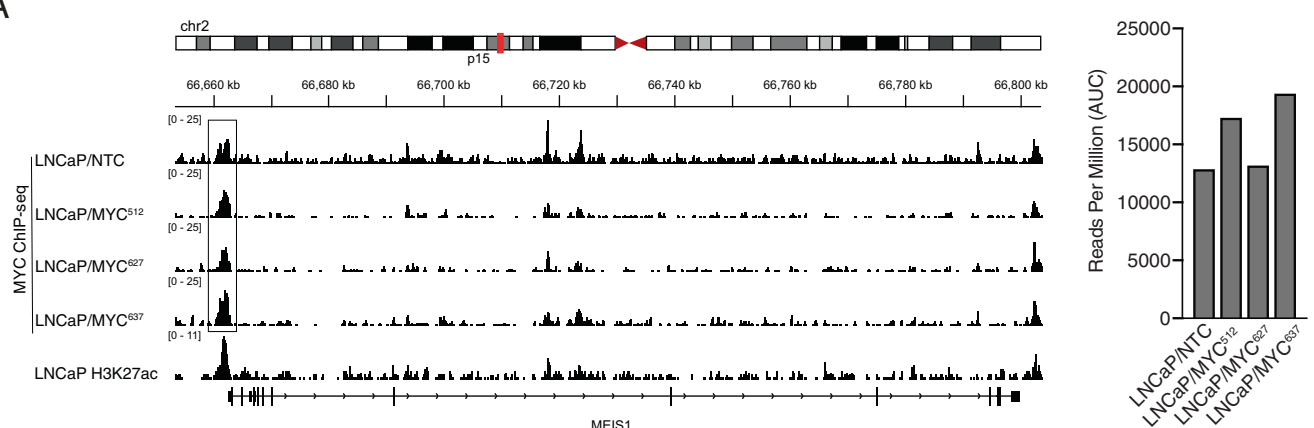

B
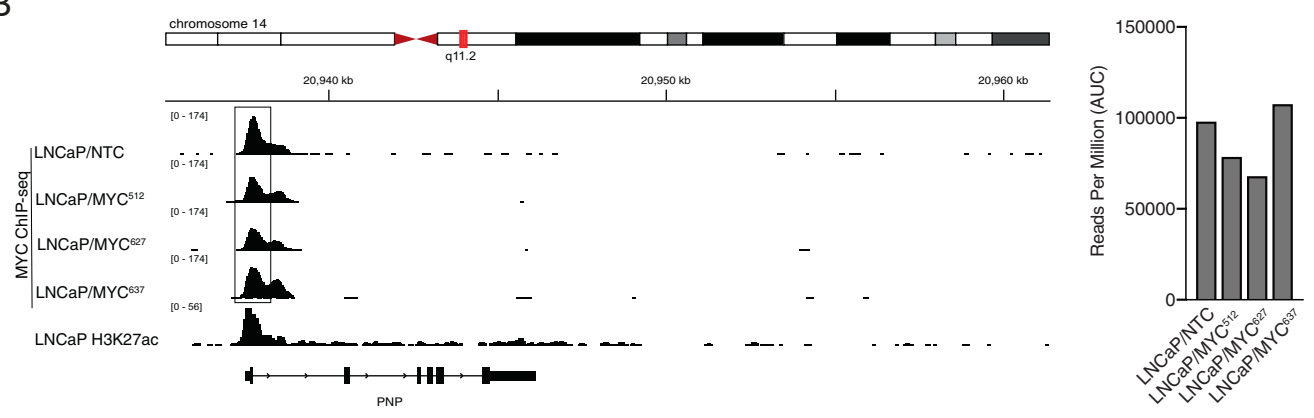

C

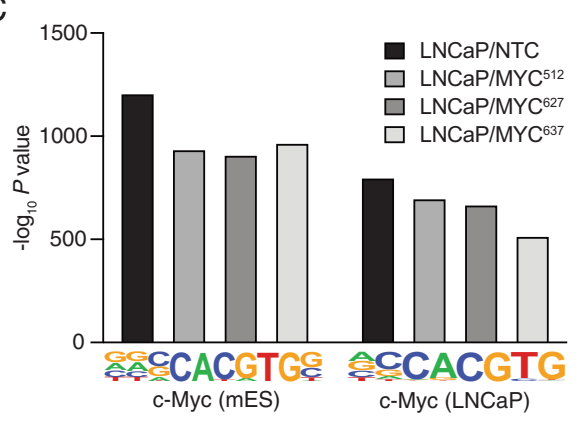

E
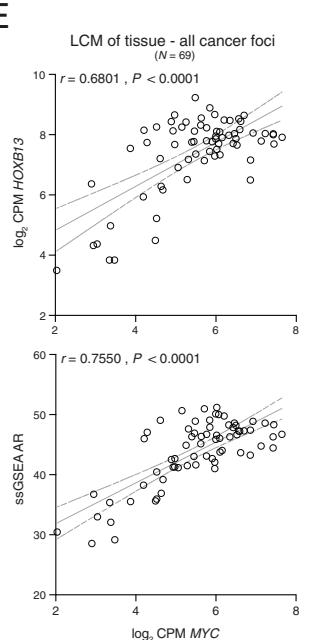

$\mathrm{F}$
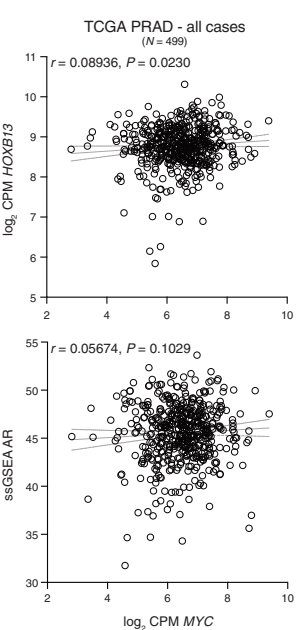

$D$

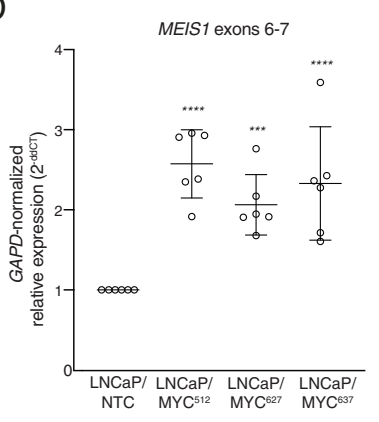

G
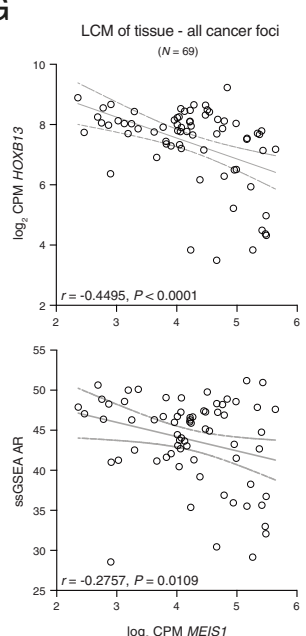

MEIS1 exons 10-12

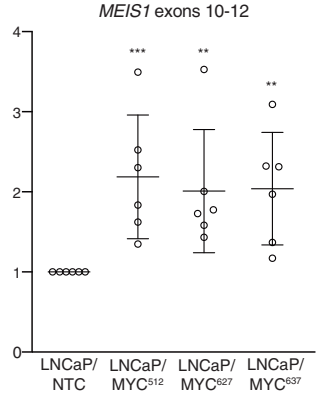

$\mathrm{H}$
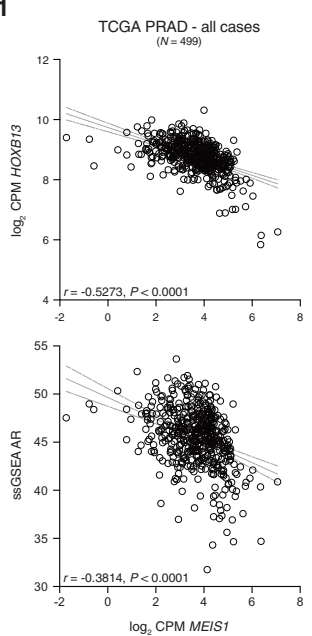

expression and AR activity in the LCM (Fig. 4g, bottom; $r=-0.2757,95 \%$ C.I. $-0.4810-0.0417, P=0.0109)$ and TCGA (Fig. 4 h, bottom; $r=-0.3814,95 \%$ C.I. -0.4540 to
$-0.3038, P<0.0001)$ cohorts. Given the potential impact of the HOXB13 G84E SNP (rs138213197) on MEIS1 interaction, expression, and function [20], we performed SNV 
Fig. 4 Decreased MYC expression increased MYC occupancy at MEISI and MEISI expression. a IGV depiction of MYC and $\mathrm{H} 3 \mathrm{~K} 27 \mathrm{ac}$ binding events in LNCaP $M Y C$ knockdown cell lines, showing increased MYC occupancy at the MEIS1 locus in cells harboring MYC knockdown. b IGV depiction of MYC and H3K27ac binding events in LNCaP $M Y C$ knockdown cell lines, showing decreased MYC occupancy at the PNP locus in cells harboring MYC knockdown. Graphs to the right of $(\mathbf{a}, \mathbf{b})$ depict the area under the curve (AUC) of read counts for the peaks outlined by the boxes. $\mathbf{c}$ The confidence of identifying known MYC binding motifs as identified by HOMER are shown for the LNCaP MYC knockdown cell lines, measured by $-\log _{10} P$ value for enrichment. $Q$ values (adjusting for false discovery) for these enrichments are zero. d Quantitative reversetranscription PCR of GAPD-normalized MEIS1 transcript in LNCaP MYC knockdown cell lines relative to control, measuring across the splice boundary of exons 6-7 (left) or exons 10-12 (right). Bars and whiskers represent the mean \pm standard deviation of six independent experiments conducted in triplicate, plotted individually as open circles $(* * P<0.01 ; * * * P<0.001 ; * * * * P<0.0001$ by Student's $t$ test). e, $\mathbf{f}$ Correlation of the $\log _{2}$ CPM expression level for $M Y C$ with the $\log _{2}$ CPM expression level for $H O X B 13$ (top) and the 266-gene ssGSEA AR activity signature score (bottom) in the entire LCM cohort $(N=69, \mathbf{e})$ and the entire TCGA PRAD cohort $(N=499, \mathbf{f})$. The gray dotted lines represent the $95 \%$ confidence intervals for each correlation. $\mathbf{g}$, h Correlation of the $\log _{2}$ CPM expression level for MEIS1 with the $\log _{2}$ CPM expression level for HOXB13 (top) and the 266-gene ssGSEA AR activity signature score (bottom) in the entire LCM cohort $(N=69, \mathbf{g})$ and the entire TCGA PRAD cohort $(N=499$, h). The gray dotted lines represent the $95 \%$ confidence intervals for each correlation.

analysis of germline samples from the LCM and TCGA cohorts (data not shown). However, none of the LCM cases carried the G84E SNP, and only five TCGA cases which carried the SNP (CH-5748, CH-5771, G9-6370, KK-A8IG, and V1-A9OF), were not amongst the MYC-high or MYClow group (see Fig. 3c). Taken together, our data suggest that in MYC-high tumors, PCa development is collectively mediated by increased AR activity and $H O X B 13$ expression resulting from MEIS1 down-regulation.

\section{Discussion}

In many cancer types, the role of amplified MYC in mediating tumorigenesis has been linked to genes involved in ribosomal biogenesis, universally up-regulated transcription, proliferation, and reprogramming cells to a pluripotent state [40]. A subset of advanced prostate cancers also harbor amplified MYC, but it is distinct from the up-regulated MYC that is a hallmark of many localized prostate cancers [3, 14-16]. In the current study, we used transcriptome profiling to assess subpopulations of prostate tumors based on differential MYC protein expression and MYC activity, and we similarly compared differentially expressed genes and pathways within the larger prostate TCGA cohort based on MYC activity. Finding that increased MYC activity was inversely proportional to overall transcription, we focused on down-regulated pathways, identifying a negative correlation between MYC activity and MEISI expression, with MYC directly involved in the negative regulation of MEISI as demonstrated by knockdown and chromatin immunoprecipitation analyses. The inverse association between MEIS1 expression and AR extended further to $H O X B 13$ expression, indicating that in a subset of primary $\mathrm{PCa}$, decreased expression of MEIS1 may be necessary for AR and HOXB13 to drive tumor development and progression.

From its discovery as an oncogene to the subsequent challenges associated with targeting MYC pharmacologically, efforts have shifted in identifying targetable MYC effector genes or other targetable co-factors that are necessary for MYC activity [41, 42]. Efforts to dissect functions of MYC have frequently relied on cancer models in which MYC levels rise up to 20-fold, in contrast to 1-2 fold physiological elevation of MYC expression in $\mathrm{PCa}$ $[2,6,43]$. Not surprisingly, the phenotypes associated with $M Y C$ up-regulation differ. For example, MYC expression in the activation of lymphocytes [26] or in Burkitt's lymphoma [27] is associated with a universal amplification of transcription, while we observed that in LNCaP PCa cells with up-regulated $M Y C$, knocking down $M Y C$ by $<50 \%$ with shRNA consistently increased the total amount of RNA produced per cell.

The differences in MYC function in PCa extend to the long-standing relationship between MYC and proliferation [40]. Here, we report a series of PCa tissues, seriallysectioned and stained with anti-MYC and anti-Ki67 antibodies, that show a weak proportional relationship. While up to $50 \%$ of luminal PCa cells were positive for MYC expression, proliferation measured by Ki67 affected $<5 \%$ of cells. We show a similar relationship in the prostate TCGA cohort and PCa cell lines, suggesting that while proliferating cells may harbor MYC activity, MYC alone is not sufficient for proliferation in PCa. This is generally in agreement with findings that MYC expression alone is not reflective of an increased proliferative fraction of $\mathrm{PCa}$ cells [5].

A key finding from our study is the negative relationship between MYC activity and MEISI expression. In the context of MYC's role as a universal transcriptional amplifier, a target gene of MYC-mediated repression could simply be considered a technical anomaly reflecting unequal numbers of cells used for analysis [26]. However, we show that transcriptional signatures of MYC activity and MEISI are inversely correlated in two large independent cohorts, which is largely consistent with the finding that tumors and PCa cells with increased MEIS1 expression show decreased enrichment of MYC target gene sets by GSEA [19]. Importantly, we further demonstrate here with ChIP-seq that the effects on MEISI expression are due in part to increased MYC occupancy at the MEISI locus, such that by using 
shRNA to lower the level MYC with biological variability observed in patient samples, it resulted in repositioning of MYC at specific sites. However, the intensity of MYC binding at MEISI, as measured by the peak height in IGV, is substantially less than the peak heights of known direct targets of MYC such as PNP, NOLC1 and EIF5A, and the increased expression of MEISI in the context of decreased $M Y C$ expression precludes $M Y C$ as a direct transcriptional activator. Indeed, MEISI is a direct target of MIZ-1 [44], which functions to increase the expression of MEIS1 in the absence of MYC. Therefore, one possible explanation for our observations is that a MYC-regulated transcriptional repressor also sits at the MEIS1 promoter and competes with MYC for direct binding to MIZ-1 or another positive regulator of MEISI transcription. In such a model, MYC would only be bound to MEISI indirectly, and the interaction between MYC/MIZ-1 complexes and other transcriptional regulators of MEIS1 is of interest for future study.

Previously, Bhanvadia et al. [19]. demonstrated that tumors with increased MEIS1 are potentially less aggressive, based on studies of LAPC-4 PCa cells expressing shRNA against MEIS1 and that tumors with lower levels of MEIS1 were at greater risk of biochemical recurrence and metastasis. Specifically, they reported a step-wise decrease in MEIS1 expression during $\mathrm{PCa}$ progression, but that knockdown of both MEIS1 and MEIS2 were necessary to suppress tumor development in vivo. HOXB13, a homeodomain transcription factor, has been shown to regulate AR activity while shRNA against HOXB13 in LAPC4 cells inhibits their growth [18]. Based on these prior findings, as HOXB13 physically interacts with MEIS1 [18], tumors expressing less MEIS1 would be expected to display greater HOXB13 expression and AR activity. Indeed, we show an inverse relationship between MEISl expression and AR activity in two independent cohorts, which is consistent with previous observations of a positive correlation between MYC expression and AR activity [7]. However, we did not observe a statistically significant inverse relationship between MYC and MEIS2 (see Supplementary Table 2).

Our study has an important limitation in that we did not assess the relationship between MYC and MEISI in additional human cell line or mouse model systems. Our focus on increased MYC activity in locoregional $\mathrm{PCa}$ is distinct from highly amplified MYC in metastatic PCa. The LNCaP model is the only commercially available PCa cell line that is genomically similar to localized PCa, derived from a regional lymph node, and is both androgen-dependent and luminal in origin. Mouse models with robustly overexpressed MYC and all other PCa cell lines derived from advanced metastases exhibit a high proliferative index, which does not accurately model early $\mathrm{PCa}$, which is a slow-growing disease.

In summary, our analysis of MYC-expressing prostate tumors demonstrates an inverse relationship with MEISI expression, which in turn is negatively correlated with HOXB13 expression and AR activity. Mechanistically, our data demonstrate that MEISI is a directly repressed target of MYC, and via effects on $H O X B 13$ link MYC activity to AR activity. The potential clinical significance of the inverse MYC/MEIS1 relationship warrants further investigation as AR-directed therapies are introduced earlier in the clinical course of disease, and MEISI levels may indicate potential sensitivity to treatment.

\section{Materials and methods}

\section{Study approval}

This research was conducted in accordance with the principles of the Declaration of Helsinki. All patients provided informed consent prior to participating in tissue procurement protocols, and all samples were deidentified as per institutional policies. The collection of radical prostatectomy specimens was approved by the Dana Farber/Harvard Cancer Center Institutional Review Boards, under protocol numbers 11-250, 15-008, and 15-492. The collection of prostate biopsy specimens was approved by the National Institutes of Health Institutional Review Board, under protocol number 15-c0124. Tissues were fixed in formalin and embedded in paraffin according to standard procedures.

\section{Pan-cancer analysis}

Gene-level normalized FPKM expression values for The Cancer Genome Atlas (TCGA) RNA-seq data were downloaded from the NCI Genomic Data Commons (https://gdc.ca ncer.gov). Cases were filtered for tumor samples within each organ type and cases with missing gene expression values were removed. Single-sample gene set enrichment analysis (ssGSEA) was performed on the GenePattern server (https:// cloud.genepattern.org) using the ssGSEA Projection module version 9.1.1 with the following parameters: weighting exponent: 0.75 ; combine mode: combine.add; sample normalization method: none. For each TCGA tumor type, ssGSEA projection values were obtained for a 17-gene MYCindependent proliferation signature, a 54-gene MYC activity signature [22], and a prostate cancer-specific gene set generated by MYC overexpression in LNCaP cells [11]. The genes used for analysis are given in Supplementary Table 1.

\section{Cell lines and derivatives}

RNA knockdown of $M Y C$ expression was achieved using the SMARTvector lentiviral shRNA system (Dharmacon) and a standard second-generation packaging system. hCMV-Turbo GFP targeting vectors were MYC 512: V3SH11240-2251 
90433 (GGTCGATGCACTCTGAGGC, targeting ORF); M YC 627: V3SH11240-226339523 (TTGATCATGCATTTG AAAC, targeting $3^{\prime}$ UTR); MYC 637: V3SH11240-2264 39183 (GTAGAAATACGGCTGCACC, targeting ORF); and non-targeting control: VSC11707. Briefly, 293FT cells (ThermoFisher) were reverse-transfected with a lentiviral shRNA expression vector in a $10 \mathrm{~cm}$ dish, using envelope (pCMV-VSV-G, \#8454, Addgene) and packaging (pCMVdR8.2 dvpr, \#8455, Addgene) vectors following the ThermoFisher ViraPower protocols. Media containing lentivirus were harvested two days post-transfection, passed through a $0.45 \mu \mathrm{m}$ filter, aliquoted and frozen. HT-1080 cells (ATCC) were used for titering virus following the ThermoFisher ViraPower protocol.

LNCaP cells (clone FGC, catalog number CRL1740) were purchased from ATCC. Cell line authentication by STR profiling and mycoplasma testing was performed every 6 months (Laragen, Inc). LNCaP cells were seeded in 6 -well plates at $1 \times 10^{5}$ cells per well and transduced with lentivirus at a multiplicity of infection of 1 in complete media for $24 \mathrm{~h}$. Complete media were then replaced one day post-transduction, and selection with puromycin $(1 \mu \mathrm{g} / \mathrm{mL}$, Life Technologies) began 2 days post-transduction. Transduction was confirmed by visual confirmation of GFP fluorescence in $>90 \%$ of cells, and knockdown was confirmed by qPCR and Western blotting.

\section{Statistical analysis}

Statistical analyses were performed using GraphPad Prism 8 for Mac. Statistical tests used and relevant variables are indicated in the legend of each figure.

Additional methods including qPCR, western blotting, bioinformatic analyses, histology and ChIP-seq are provided in Supplementary Information.

\section{Data availability}

Human tissue RNA-seq data has been deposited into the Database of Genotypes and Phenotypes (https://dbgap.ncbi. nlm.nih.gov/), accession ID phs001813.v1.p1. Human tissue gene expression data has been deposited into the Gene Expression Omnibus (https://www.ncbi.nlm.nih.gov/geo/), accession ID GSE130046. ChIP-seq data has been deposited into the Sequence Read Archive (https://www. ncbi.nlm.nih.gov/sra), accession ID SRP218384 and GEO, accession ID GSE135942.

\section{Code availability}

The code used to perform all analyses is available at https:// github.com/CBIIT/lgcp.
Acknowledgements The authors gratefully acknowledge the patients and the families of patients who contributed to this study. RNA-seq and ChIP-seq was performed at the CCR Illumina Sequencing Facility. The authors acknowledge technical assistance from Carla Calagua. Portions of this research utilized the computational resources of the NIH HPC Biowulf cluster.

Funding This work was supported by the Prostate Cancer Foundation (Young Investigator Awards to SW, HY and AGS) and the Intramural Research Program of the NIH, National Cancer Institute.

Author contributions Design of the study: NCW, BEG, BJC, AGS; Tissue collection and pathology: HY; Immunohistochemistry and microdissection: NCW, SYT, SW, RL, NVC, RA, EDW; Data collection: NCW, NTT, STH, RL, NVC, RA, Library preparation and NGS: NCW, SYT; Data analysis: NCW, SYT, NTT, BEG, BJC, HY, AGS; Paper preparation: NCW, AGS.

\section{Compliance with ethical standards}

Conflict of interest The authors declare that they have no conflict of interest.

Publisher's note Springer Nature remains neutral with regard to jurisdictional claims in published maps and institutional affiliations.

Open Access This article is licensed under a Creative Commons Attribution 4.0 International License, which permits use, sharing, adaptation, distribution and reproduction in any medium or format, as long as you give appropriate credit to the original author(s) and the source, provide a link to the Creative Commons license, and indicate if changes were made. The images or other third party material in this article are included in the article's Creative Commons license, unless indicated otherwise in a credit line to the material. If material is not included in the article's Creative Commons license and your intended use is not permitted by statutory regulation or exceeds the permitted use, you will need to obtain permission directly from the copyright holder. To view a copy of this license, visit http://creativecommons. org/licenses/by/4.0/.

\section{References}

1. Cancer Genome Atlas Research Network. The molecular taxonomy of primary prostate cancer. Cell. 2015; 163:1011-25.

2. Taylor BS, Schultz N, Hieronymus H, Gopalan A, Xiao Y, Carver $\mathrm{BS}$, et al. Integrative genomic profiling of human prostate cancer. Cancer Cell. 2010;18:11-22.

3. Sowalsky AG, Kissick HT, Gerrin SJ, Schaefer RJ, Xia Z, Russo $\mathrm{JW}$, et al. Gleason score 7 prostate cancers emerge through branched evolution of clonal gleason pattern 3 and 4. Clin Cancer Res. 2017;23:3823-33.

4. Trock BJ, Fedor H, Gurel B, Jenkins RB, Knudsen BS, Fine SW, et al. PTEN loss and chromosome 8 alterations in Gleason grade 3 prostate cancer cores predicts the presence of un-sampled grade 4 tumor: implications for active surveillance. Mod Pathol. 2016;29:764-71.

5. Gurel B, Iwata T, M Koh C, Jenkins RB, Lan F, Van Dang C, et al. Nuclear MYC protein overexpression is an early alteration in human prostate carcinogenesis. Mod Pathol. 2008;21:1156. (Original Article)

6. Koh CM, Bieberich CJ, Dang CV, Nelson WG, Yegnasubramanian S, De Marzo AM. MYC and prostate cancer. Genes Cancer. 2010;1:617-28. 
7. Bai S, Cao S, Jin L, Kobelski M, Schouest B, Wang X, et al. A positive role of c-Myc in regulating androgen receptor and its splice variants in prostate cancer. Oncogene. 2019;38:4977-89.

8. Packer JR, Maitland NJ. The molecular and cellular origin of human prostate cancer. Biochimica et Biophysica Acta. 1863;2016:1238-60.

9. Norris JD, Chang CY, Wittmann BM, Kunder RS, Cui H, Fan D, et al. The homeodomain protein HOXB13 regulates the cellular response to androgens. Mol Cell. 2009;36:405-16.

10. Yang YA, Yu J. Current perspectives on FOXA1 regulation of androgen receptor signaling and prostate cancer. Genes Dis. 2015;2:144-51.

11. Barfeld SJ, Urbanucci A, Itkonen HM, Fazli L, Hicks JL, Thiede $\mathrm{B}$, et al. c-Myc antagonises the transcriptional activity of the androgen receptor in prostate cancer affecting key gene networks. EBioMedicine. 2017;18:83-93.

12. Barfeld SJ, Fazli L, Persson M, Marjavaara L, Urbanucci A, Kaukoniemi KM, et al. Myc-dependent purine biosynthesis affects nucleolar stress and therapy response in prostate cancer. Oncotarget. 2015;6:12587-602.

13. Koh CM, Gurel B, Sutcliffe S, Aryee MJ, Schultz D, Iwata T, et al. Alterations in nucleolar structure and gene expression programs in prostatic neoplasia are driven by the MYC oncogene. Am J Pathol. 2011;178:1824-34.

14. Baena-Del Valle JA, Zheng Q, Esopi DM, Rubenstein M, Hubbard GK, Moncaliano MC, et al. MYC drives overexpression of telomerase RNA (hTR/TERC) in prostate cancer. J Pathol. 2018;244:11-24.

15. Robinson D, Van Allen Eliezer M, Wu Y-M, Schultz N, Lonigro Robert J, Mosquera J-M, et al. Integrative clinical genomics of advanced prostate cancer. Cell. 2015;161:1215-28.

16. Sowalsky AG, Ye H, Bhasin M, Van Allen EM, Loda M, Lis RT, et al. Neoadjuvant-intensive androgen deprivation therapy selects for prostate tumor foci with diverse subclonal oncogenic alterations. Cancer Res. 2018;78:4716-30.

17. Park JW, Lee JK, Phillips JW, Huang P, Cheng D, Huang J, et al. Prostate epithelial cell of origin determines cancer differentiation state in an organoid transformation assay. Proc Natl Acad Sci USA. 2016;113:4482-7.

18. Johng D, Torga G, Ewing CM, Jin K, Norris JD, McDonnell DP, et al. HOXB13 interaction with MEIS1 modifies proliferation and gene expression in prostate cancer. Prostate. 2019;79:414-24.

19. Bhanvadia RR, VanOpstall C, Brechka H, Barashi NS, Gillard M, McAuley EM, et al. MEIS1 and MEIS2 expression and prostate cancer progression: a role for HOXB13 binding partners in metastatic disease. Clin Cancer Res. 2018;24:3668-80.

20. Brechka H, Bhanvadia RR, VanOpstall C, Vander Griend DJ. HOXB13 mutations and binding partners in prostate development and cancer: function, clinical significance, and future directions. Genes Dis. 2017;4:75-87.

21. Chen H, Liu H, Qing G. Targeting oncogenic Myc as a strategy for cancer treatment. Signal Transduct Target Ther. 2018;3:5.

22. Shaffer AL, Wright G, Yang L, Powell J, Ngo V, Lamy L, et al. A library of gene expression signatures to illuminate normal and pathological lymphoid biology. Immunological Rev. 2006;210:67-85.

23. Hammarsten P, Josefsson A, Thysell E, Lundholm M, Hagglof C, Iglesias-Gato $\mathrm{D}$, et al. Immunoreactivity for prostate specific antigen and Ki67 differentiates subgroups of prostate cancer related to outcome. Mod Pathol. 2019;32:1310-9.

24. Tretiakova MS, Wei W, Boyer HD, Newcomb LF, Hawley S, Auman H, et al. Prognostic value of Ki67 in localized prostate carcinoma: a multi-institutional study of $>1000$ prostatectomies. Prostate Cancer Prostatic Dis. 2016;19:264-70.
25. Cunningham D, You Z. In vitro and in vivo model systems used in prostate cancer research. J Biol Methods. 2015;2:e17.

26. Nie Z, Hu G, Wei G, Cui K, Yamane A, Resch W, et al. c-Myc is a universal amplifier of expressed genes in lymphocytes and embryonic stem cells. Cell. 2012;151:68-79.

27. Lin CY, Lovén J, Rahl PB, Paranal RM, Burge CB, Bradner JE, et al. Transcriptional amplification in tumor cells with elevated cMyc. Cell. 2012;151:56-67.

28. Junttila MR, Westermarck J. Mechanisms of MYC stabilization in human malignancies. Cell Cycle. 2008;7:592-6.

29. Hawksworth D, Ravindranath L, Chen Y, Furusato B, Sesterhenn IA, McLeod DG, et al. Overexpression of C-MYC oncogene in prostate cancer predicts biochemical recurrence. Prostate Cancer Prostatic Dis. 2010;13:311. (Original Article)

30. Marcos S, González-Lázaro M, Beccari L, Carramolino L, MartinBermejo MJ, Amarie O, et al. Meis1 coordinates a network of genes implicated in eye development and microphthalmia. Development. 2015;142:3009.

31. Wang GG, Pasillas MP, Kamps MP. Meis1 programs transcription of FLT3 and cancer stem cell character, using a mechanism that requires interaction with $\mathrm{Pbx}$ and a novel function of the Meis1 Cterminus. Blood. 2005;106:254-64.

32. Calvo KR, Sykes DB, Pasillas MP, Kamps MP. Nup98-HoxA9 immortalizes myeloid progenitors, enforces expression of Hoxa9, Hoxa7 and Meis1, and alters cytokine-specific responses in a manner similar to that induced by retroviral co-expression of Hoxa9 and Meis1. Oncogene. 2002;21:4247-56.

33. Takeda A, Goolsby C, Yaseen NR. NUP98-HOXA9 induces longterm proliferation and blocks differentiation of primary human CD34 + hematopoietic cells. Cancer Res. 2006;66:6628-37.

34. Collins CT, Hess JL. Deregulation of the HOXA9/MEIS1 axis in acute leukemia. Curr Opin Hematol. 2016;23:354-61.

35. Yohe ME, Gryder BE, Shern JF, Song YK, Chou H-C, Sindiri S, et al. MEK inhibition induces MYOG and remodels super-enhancers in RAS-driven rhabdomyosarcoma. Sci Transl Med. 2018;10:eaan4470.

36. Gallenne T, Ross KN, Visser NLS, Desmet CJ, Wittner BS, et al. Systematic functional perturbations uncover a prognostic genetic network driving human breast cancer. Oncotarget. 2017; 8:20572-87.

37. Dunham I, Kundaje A, Aldred SF, Collins PJ, Davis CA, Doyle F, et al. An integrated encyclopedia of DNA elements in the human genome. Nature. 2012;489:57-74.

38. Cui L, Li M, Feng F, Yang Y, Hang X, Cui J, et al. MEIS1 functions as a potential AR negative regulator. Exp Cell Res. 2014;328:58-68.

39. Mendiratta P, Mostaghel E, Guinney J, Tewari AK, Porrello A, Barry WT, et al. Genomic strategy for targeting therapy in castration-resistant prostate cancer. J Clin Oncol. 2009;27: 2022-9.

40. Dang CV. MYC on the path to cancer. Cell. 2012;149:22-35.

41. Dang CV. c-Myc target genes involved in cell growth, apoptosis, and metabolism. Mol Cell Biol. 1999;19:1-11.

42. Delmore JE, Issa GC, Lemieux ME, Rahl PB, Shi J, Jacobs HM, et al. BET bromodomain inhibition as a therapeutic strategy to target c-Myc. Cell. 2011;146:904-17.

43. Neiman PE, Ruddell A, Jasoni C, Loring G, Thomas SJ, Brandvold KA, et al. Analysis of gene expression during myc oncogeneinduced lymphomagenesis in the bursa of Fabricius. Proc Natl Acad Sci USA. 2001;98:6378-83.

44. Varlakhanova N, Cotterman R, Bradnam K, Korf I, Knoepfler PS. Myc and Miz-1 have coordinate genomic functions including targeting Hox genes in human embryonic stem cells. Epigenetics Chromatin. 2011;4:20. 